\title{
El último descubrimiento anatómico: las glándulas paratiroides - Glandulae parathyreoideae de Owen-Sandström-Gley
}

\author{
The latest anatomical discovery: the parathyroid glands or Glandulae parathyreoideae of \\ Owen-Sandström-Gley
}

Carlos Ortiz-Hidalgo*

Departamento de Anatomía Patológica, Hospital y Fundación Médica Sur, Ciudad de México, Mexico

\begin{abstract}
Resumen
En 1852, Owen, un destacado anatomista británico, describió las glándulas paratiroides. Mientras realizaba la disección de un rinoceronte indio, observó un pequeño cuerpo amarillo compacto, unido a la tiroides. Virchow, y más tarde Remak, describieron las paratiroides humanas alrededor de 1860, pero el crédito por la primera descripción completa es para Sandström en 1880. Más de una década después, Gley demostró que era la eliminación de las paratiroides lo que explicaba la tetania después de la tiroidectomía. La asociación de la patología paratiroidea y las anomalías esqueléticas fue establecida en 1914 por Erdheim y Schlagenhaufer, y Mandl fue el primer cirujano en tratar con éxito un caso de osteítis fibrosa mediante la extirpación quirúrgica de un adenoma paratiroideo en 1925. El trabajo más extenso sobre el hiperparatiroidismo fue realizado en la década de 1930 por Albright, en Boston, quien describió la hiperplasia paratiroidea y la diferenció del hiperparatiroidismo primario, secundario y terciario. Los avances en anatomía, fisiología y cirugía de las glándulas paratiroides han contribuido a diversas modalidades efectivas de diagnóstico y tratamiento.
\end{abstract}

Palabras clave: Paratiroides. Historia. Richard Owen. Ivar Sandström. Marcel Eugène Émile Gley.

\begin{abstract}
In 1852, Owen, a prominent British anatomist, described the parathyroid glands. While dissecting a rhinoceros, he noted a small compact yellow body, attached to the thyroid. Virchow and later Remak described the human parathyroids around 1960, but credit for the first complete description goes to the Sandström in 1980. More than a decade later Gley, showed that it was the removal of the parathyroids that accounted for the tetany that followed thyroidectomy. The association of parathyroid pathology and skeletal abnormalities was made in 1914 by Erdheim and Schlagenhaufer, and Mandl, was the first surgeon to successfully treat a case of osteitis fibrosa by surgical removal of a parathyroid adenoma in 1925. The most extensive work on hyperparathyroidism was done in the 1930s by Albright form Boston, who described parathyroid hyperplasia, and differentiated between primary, secondary and tertiary hyperparathyroidism. Progresses in anatomy, physiology and surgery of the parathyroid glands, have contributed to various effective modalities of diagnosis and treatment.
\end{abstract}

Keywords: Parathyroids. History. Richard Owen. Ivar Sandström. Marcel Eugène Émile Gley.

\author{
Correspondencia: \\ ${ }^{*}$ Carlos Ortiz-Hidalgo \\ Avda. Puente de Piedra, 150 \\ Col. Toriello Guerra, Del. Tlalpan \\ Fecha de recepción: 26-11-2020 \\ Cir Cir. 2022;90(1):140-149 \\ C.P. 14050, Ciudad de México, México \\ Fecha de aceptación: 15-01-2021 \\ DOI: $10.24875 / C I R U .20001307$ \\ Contents available at PubMed \\ E-mail: ortizhidalgocarlos@gmail.com \\ 0009-7411/@ 2021 Academia Mexicana de Cirugía. Publicado por Permanyer. Este es un artículo open access bajo la licencia CC BY-NC-ND \\ www.cirugiaycirujanos.com \\ (http://creativecommons.org/licenses/by-nc-nd/4.0/).
}


"Is seems hardly credible that the loss of bodies (parathyroids) so tiny, should be followed by a result so disastrous."

William S. Halsted (1852-1922)

\section{Introducción}

Las glándulas paratiroides son esenciales para la vida, pues desempeñan un papel central en el metabolismo del calcio. Su pequeño tamaño (entre 3 y $7 \mathrm{~mm}$ ) no va en proporción con su importante función en la homeostasis. Es posible que este pequeño tamaño haya sido la causa de la demora en su descubrimiento y su subsecuente entendimiento fisiológico ${ }^{1,2}$. Las paratiroides son las glándulas más recientemente descritas en el ser humano. La forma en que fueron descubiertas y los eventos que siguieron a su identificación forman una crónica con diversos protagonistas que hicieron aportaciones trascendentales para el discernimiento del desarrollo, la histología, la función y las anomalías de las glándulas paratiroides ${ }^{3,4}$. A continuación, se relata una síntesis de la historia de estas pequeñas glándulas descritas hace poco más de 150 años.

\section{Descubrimiento de las glándulas paratiroides}

\section{Sir Richard Owen}

La primera descripción de la glándula paratiroides fue realizada el 19 de noviembre de 1849 por Sir Richard Owen (1804-1892) durante la autopsia que realizó a un rinoceronte que había muerto en el zoológico de Londres ${ }^{5}$ (Fig. 1A). Owen nació en Lancaster, Inglaterra, el 20 de julio de 1804, y comenzó a estudiar medicina en Edimburgo en 1820. Fue en esa Universidad donde aprendió a disecar cadáveres, lo que posteriormente tuvo un papel cardinal en su carrera académica. Aparentemente fueron las malas y aburridas clases de anatomía de Alexander Monro III (tertius) (1773-1859) lo que le llevó a abandonar Edimburgo y trasladarse a Londres para ingresar al St. Bartholomew's Hospital, donde se tituló como cirujano Fellow of the Royal College of Surgeons a los 23 años de edad 5 ,6. En 1834 ocupó el cargo de profesor de anatomía comparada en el St. Bartholomew's Hospital, y fue quien se encargó de ordenar toda la colección que John Hunter (1728-1793) había dejado, por lo que fue nombrado Profesor Hunteriano ${ }^{5,6}$. A la muerte de Charles Bell (1774-1842), conocido por la descripción de la parálisis facial, fue elegido profesor de anatomía y fisiología. Owen dedicó un enorme interés al estudio de la paleontología, y fue quien dio el nombre de "dinosaurios» a ese grupo de saurópsidos que aparecieron durante el Triásico (del griego

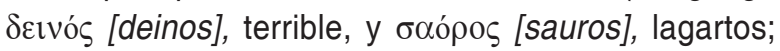
o sea, jlagartos terribles!) ${ }^{5}$. Este nombre no es del todo preciso, ya que los dinosaurios no son lagartos, pero Owen pretendía evocar su majestuosidad y formidable tamaño ${ }^{7}$.

Owen fue un escritor prolífico y su producción literaria asciende a más de 600 artículos y diversos libros, de los cuales los más notables son Lectures on Comparative Anatomy and Physiology of the Vertebrate Animals (1846), A History of British Fossil Mammals and Birds (1846), A History of British Fossil Reptiles (1849-84) y On the Anatomy of Vertebrates $(1866-68)^{8}$. En el libro Odontography (1840-45) hace un estudio minucioso sobre la estructura de los dientes y es donde describe las líneas irregulares de la dentina, hoy llamadas «líneas intercementales de Owen»6,8.

Owen supo bien consolidarse en la cúspide de la comunidad científica, pero al ser uno de los críticos más severos de Charles Darwin (1809-1882), por su abierta oposición a la teoría de la evolución, no solo perdió su buen nombre, sino que también se ganó la designación de "el gran villano de la ciencia»9. Darwin, que había sido su amigo por más de 20 años, se sintió traicionado, y se dice que fue al único científico al que el naturalista llegó a odiar ${ }^{8}$.

El 20 de septiembre de 1834, la Zoological Society de Londres compró un rinoceronte indio (Rhinoceros unicornis) por 1000 guineas (la guinea fue una moneda que se utilizó en Inglaterra, y 1000 guineas eran aproximadamente $\$ 25,249.60$ pesos mexicanos al tipo de cambio actual) y rápidamente se convirtió en la mayor atracción de zoológico de Londres ${ }^{5,6,8}$. Este animal se acomodó junto con los elefantes y no fue bien aceptado, pues uno de ellos le atacó. Poco tiempo después, posiblemente secundario al daño provocado por el elefante, el rinoceronte murió, el 19 de noviembre de 1849. Tal vez nadie imaginó que la muerte de este animal aportaría algo muy importante al conocimiento de la anatomía humana. Owen disecó al animal, de 2 toneladas, durante los meses de invierno de 1849 a 1850, y para cuando completó el trabajo Owen mencionó que «estaba ya en un estado ofensivo de descomposición» 6 .

El informe de Owen incluyó tanto los últimos días de vida del rinoceronte, de acuerdo con lo registrado 

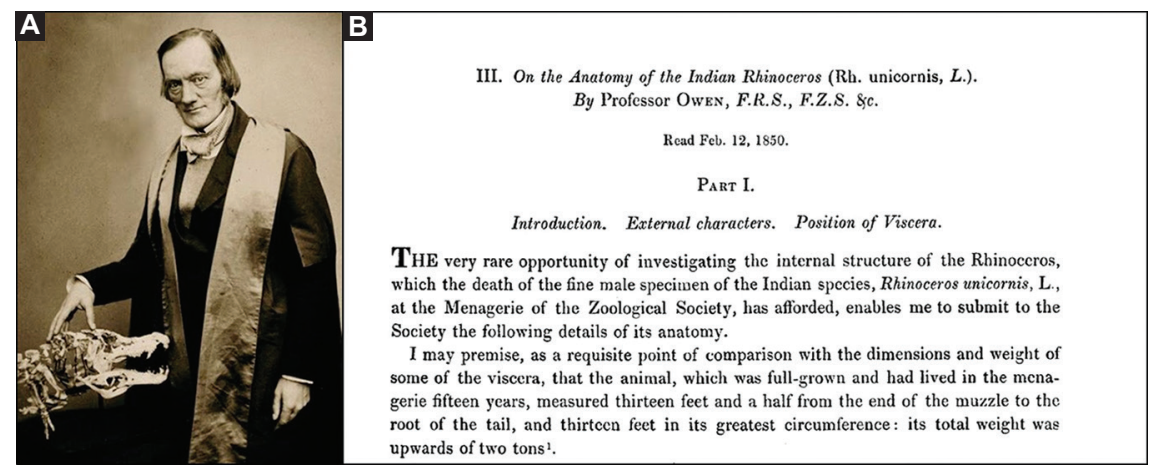

Figura 1. A: Sir Richard Owen (1804-1892). Fotografía, Maull \& Polybank. Fuente: https://wellcomeimages.org/indexplus/obf_images/1e/cc/ fb3a20c6b142ff5619e472cbc930.jpg. B: portada del trabajo de Owen donde describe las glándulas paratiroides en un rinoceronte. Fuente: Owen R. On the Anatomy of the Indian Rhinoceros (Rh. unicornis, L.). Zool Soc. 1852;IV:31-58.

en el libro de actas del cuidador en jefe del zoológico, como todo el proceso de la disección ${ }^{9}$. Así, podemos leer que el animal perdió el apetito a partir de julio 1849 y vomitó mucosidad viscosa y sangre durante una semana antes de su muerte. Owen descubrió que una costilla estaba fracturada y había lesionado el pulmón izquierdo, y concluyó que esta había sido la causa de la muerte, posiblemente secundaria al daño producido por el elefante ${ }^{8}$.

En una carta que escribió Owen a su hermana le contó:

"Amongst other matters time-devouring, and putting out of memory mundane relatives, sisters included, has been the decease of my ponderous and respectable old friend and client the rhinoceros. I call him 'client' because fifteen years ago I patronised him, and took it upon my skill, in discerning through a pretty thick hide the internal constitution, to aver that the beast would live to be a credit to the Zoological Gardens, and that he was worth the 1000 guineas demanded for him. The Council had faith, and bought him, and he has eaten their hay, oats, rice, carrots and bread in Brobdignagian quantities daily ever since, and might have gone on digesting, had he not, by some clumsy fall or otherwise inexplicable process, cracked a rib; said fracture injuring the adjacent lung and causing his demise. His anatomy will furnish forth an immortal 'Monograph', and so comfort comes to me in a shape in which it cannot he had by any of my brother Fellows"

Con el título On the anatomy of the Indian Rhinoceros (Rh. unicorni L), el 12 de febrero de 1850 Owen presentó los resultados en una monografía ante la Zoological Society (Fig. 1B) ${ }^{10}$. Este manuscrito apareció en el cuarto volumen de la revista Society's
Transactions, que abarca el periodo de enero de 1851 a septiembre de 1862. En aquellas primeras publicaciones de la Zoological Society, los documentos eran informados en conjunto e incluían varios años, por lo que el trabajo de Owen salió en el volumen fechado en 1862, pero en realidad el descubrimiento fue 10 años antes, lo cual es un dato de valor en relación con la prioridad en el descubrimiento de las glándulas paratiroides $^{5,8}$.

En el apartado III del informe titulado Víscera torácica, Owen describió: "a small compact yellow glandular body, attached to the thyroid at the point where the veins emerge»; estas corresponden a las glándulas paratiroides, pero no realizó estudio histológico ni especuló sobre función alguna de dichas estructuras $^{10}$. El espécimen que muestra la glándula paratiroidea del rinoceronte está clasificado como RCSHM/ L333.1 y se encuentra en el Museo Hunteriano, en The Royal College of Surgeons of England, en Londres. Este espécimen consiste en parte de la laringe y la tráquea del rinoceronte, y muestra en el lóbulo lateral de la tiroides el «pequeño cuerpo compacto glandular amarillo adosado a la tiroides en el punto donde emergen las venas», unido a su parte superior y parcialmente incrustado en el tejido (Fig. 2). Por todo lo anterior, las paratiroides han sido llamadas "glándulas de Owen".

Owen murió a los 88 años y es reconocido como una de las mentes más brillantes de la Inglaterra victoriana. Seguramente no imaginó que la descripción que hizo de las glándulas paratiroides de su «pesado y respetable viejo amigo y cliente, el rinoceronte" se convertiría en uno los momentos más memorables de su larga y muy fructífera vida 5 . 


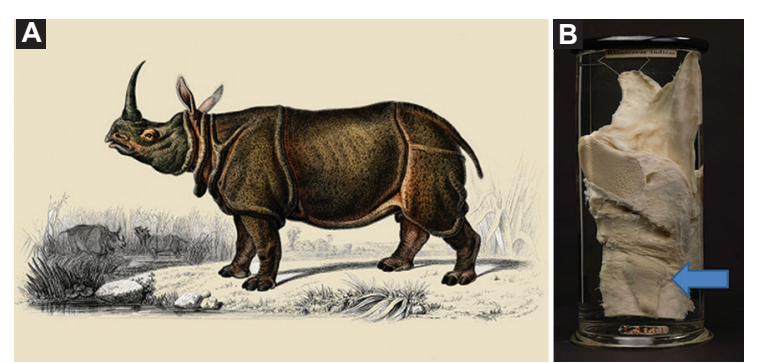

Figura 2. A: rinoceronte indio (Rhinoceros unicornis), similar al que disecó Owen. Fuente: https://www.freepik.es/vector-gratis/rinoceronte-indio-rhinoceros-unicornis_3098888.htm. B: laringe del rinoceronte donde se encuentra una glándula paratiroides (flecha) (Royal College of Surgeons of England, Mus., Physiol. Series, L 331.1).

\section{Ivar Viktor Sandström}

Tres años después de la publicación de Owen, el histólogo polaco Robert Remak (1815-1865), y posteriormente en 1863 el patólogo alemán Rudolf Ludwig Karl Virchow (1821-1902), ambos trabajando en Berlín, describieron unas glándulas similares en gatos y humanos, pero no les asignaron nombre ni especularon sobre su función ${ }^{3,4}$. No fue hasta 1880 que Ivar Viktor Sandström (1852-1889) describió su localización exacta y su estructura histológica con precisión, y propuso el nombre (Fig. 3A) ${ }^{11}$.

Sandström nació el 22 de marzo de 1852 en Estocolmo y estudió medicina en la Universidad de Uppsala. Hacia 1877, cuando acababa de cumplir los 25 años y era aún estudiante de medicina, efectuó la primera descripción anatómica e histológica de las glándulas paratiroides durante la disección del cuello de un perro en el departamento de anatomía de la Universidad de Uppsala ${ }^{3,11}$. El trabajo en que describió sus hallazgos fue escrito en alemán y enviado a la revista editada por Virchow para su publicación, pero fue rechazado porque, según le informaron, jtenía una extensión excesiva! Existe la creencia de que como Virchow ya había visto algunas estructuras similares a las descritas por Sandström, no estuvo dispuesto a darle la prioridad del descubrimiento al joven sueco, y fue por esto que rechazó el artículo ${ }^{11}$. Finalmente, el manuscrito fue publicado en sueco en 1880 en la revista Upsala Läkareförenings Förhandlingar, con el título Om en ny körtel hos menniskan och åtskilliga däggdjur (Sobre una nueva glándula en el hombre y algunos mamíferos) $)^{3,12,13}$. En este artículo, que costa de 30 páginas, Sandström indicó:

«Hace unos tres años encontré en la glándula tiroides de un perro un órgano pequeño, apenas del tamaño de una semilla de cáñamo, que estaba dentro del tejido conectivo de la tiroides, pero podía distinguirse por un color más claro. El examen microscópico reveló tejido glandular totalmente diferente al de la tiroides y con una rica vascularización." (Figs. 3 B-C $)^{12}$.

Sandström llamó a estas estructuras glandulae parathyroidea, y supuso que eran porciones embrionarias no desarrolladas de la glándula tiroides. Posteriormente disecó gatos, conejos y humanos, y encontró que había glándulas similares. Cuidadosamente disecó 50 autopsias y en 43 de ellas encontró cuatro glándulas, dos a cada lado de la tiroides, y elaboró una descripción detallada sobre la posición, el tamaño, el color y las diversas formas de las glándulas, y mostró que siempre se encontraban en la proximidad de la tiroides y medían de 3 a $15 \mathrm{~mm}$ de diámetro. Señaló que:

«La existencia de una glándula hasta ahora desconocida en animales que tan a menudo han sido un tema del examen anatómico, requirió un enfoque exhaustivo de la región alrededor de la glándula tiroides incluso en el hombre. Aunque la probabilidad de encontrar algo hasta ahora no reconocido parecía tan poco probable que fue exclusivamente con el propósito de completar la investigación, en vez de con la esperanza de encontrar algo nuevo, que comencé el examen cuidadoso de esta región. Fue tan grande mi asombro, cuando en el primer individuo examinado encontré en ambos lados, en el borde inferior de la glándula tiroides, un órgano del tamaño de un pequeño guisante, que, a juzgar por su exterior, no parecía ser una glándula linfática, ni una glándula tiroides accesoria, y al examen histológico mostró una estructura peculiar ${ }^{12}$.

A Sandström le fueron otorgadas dos medallas médicas suecas y fue invitado a presentar sus hallazgos ante la Sociedad de Ciencias Naturales en Estocol$\mathrm{mo}^{11}$. Sin embargo, su descubrimiento en ese momento no tuvo mucha repercusión. Su prometedora carrera de investigador fue truncada por una tragedia, pues por problemas depresivos crónicos se suicidó a la corta edad de 37 años $^{14}$. No obstante, la originalidad y la precisión de su descubrimiento le han asegurado por siempre un lugar en la historia de la medicina. A decir de R.L Thomson, «este artículo de Sandström es tan minucioso que poco se ha agregado a nuestro conocimiento desde su descubrimiento, y es tan completo como para no dejar ninguna duda que bien merece todo el crédito por la descripción de estos órganos» ${ }^{15}$. Por ello, las glándulas paratiroides también han sido llamadas "glándulas de Sandström». 

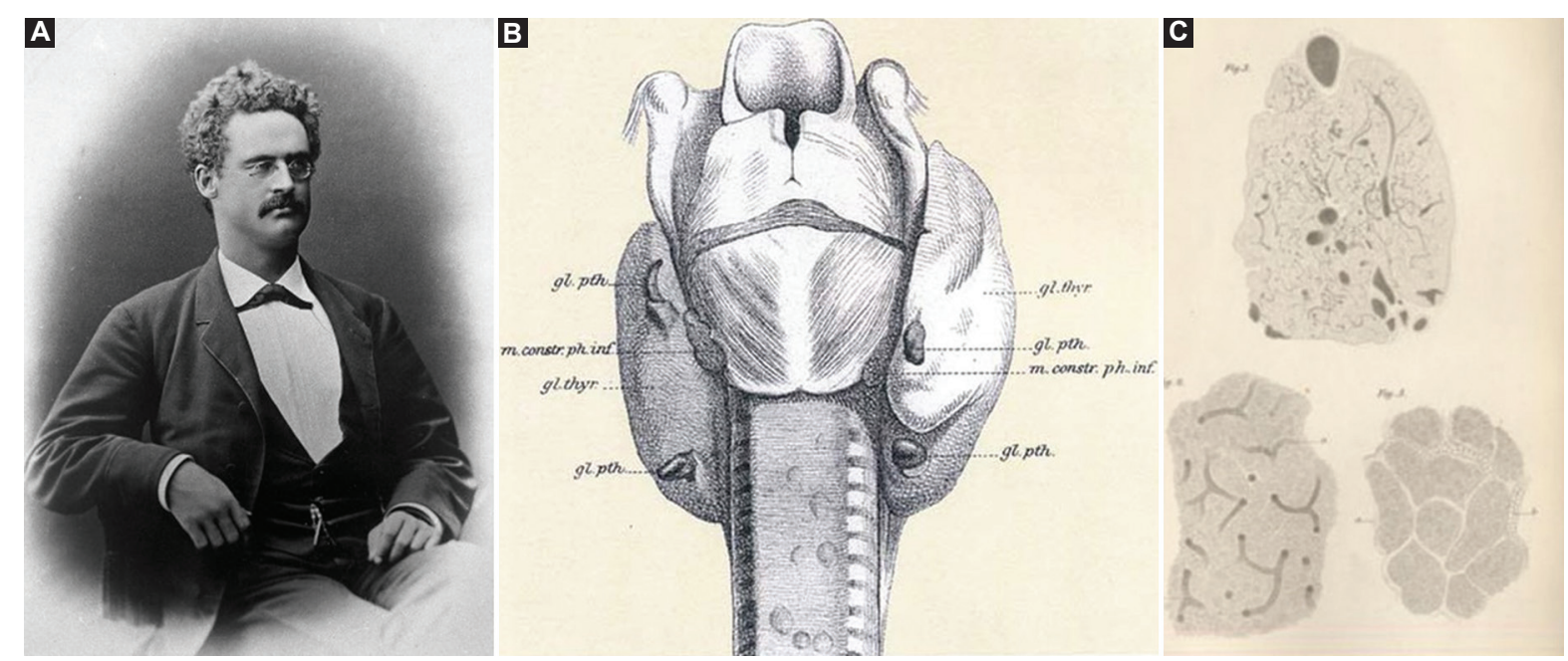

Figura 3. A: Ivar Viktor Sandström. B: dibujo que ilustra la localización anatómica de las paratiroides. C: primeros dibujos de la histología de las glándulas paratiroides. Fuente: Sandström IV. On a new gland in man and several mammals - glandulae parathyroideae. Upsala Läk Förenings Förh. 1879-80;15:441-71. Fuente: https://wellcomeimages.org/indexplus/obf_images/c9/d5/ace8e8ed53e91666002e726e2c00.jpg.

\section{Aportaciones al estudio de la paratiroides después de Owen y Sandström}

Casi una década después de la publicación de Sandström, el fisiólogo y endocrinólogo francés del Hôtel-Dieu, Marcel Eugène Émile Gley (1857-1930), quien fuera sucesor de Charles-Édouard Brown-Séquard (1817-1894) en el Collège de France, descubrió la importancia de estas glándulas y publicó el primer informe sobre la relación entre las glándulas paratiroides y la tetania (Fig. 4) 3,4,11.

Gley nació en la ciudad de Épinal, situada a 400 km al este de Paris, y estudió fisiología en Nancy. Posteriormente se trasladó a París, donde recibió el título de Professeur agrégé, y en 1908 se convirtió en profesor en el Collège de France. Gley advirtió, durante algunos experimentos realizados en perros, que estos presentaban tetania después de la destrucción inadvertida de las paratiroides durante la extirpación de la glándula tiroides. Demostró que cuando se retiraban las glándulas paratiroides de los animales de laboratorio, estos presentaban muertes por tetania, y pensó, erróneamente, que las glandules thyroidiennes, como él se refería a ellas, completaban de alguna forma la función de la tiroides ${ }^{3,4}$. No pudo darse cuenta de que estas glándulas tenían un papel totalmente diferente. No obstante esta mala interpretación, Gley había encontrado una función elemental de estas glándulas y, debido a esto, en ocasiones también han sido también llamadas "glándulas de Gley»3,4.

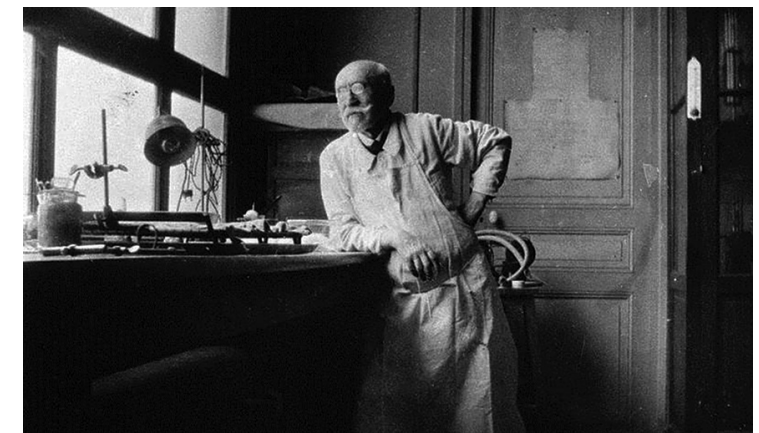

Figura 4. Marcel Eugène Émile Gley (1857-1930) en su laboratorio del Hôtel-Dieu, en Paris. Fuente: https://wellcomeimages.org/indexplus/ image/V0026444.html.

Basado en los estudios de Gley, William George MacCallum (1874-1944), entonces jefe del departamento de patología del Hospital Johns Hopkins, describió que la tetania era directamente el resultado de la deficiencia de calcio, y por la misma época se demostró que la tetania por hipocalcemia podría ser tratada con inyecciones de extracto de paratiroides ${ }^{16}$.

La relación que tienen las paratiroides con la enfermedad ósea fue inicialmente descrita por el patólogo del Instituto Patológico de Estrasburgo, Friedrich Daniel von Recklinghausen (1833-1910). En 1891, para festejar el 71 cumpleaños de Virchow, 13 de sus alumnos, entre los que figuran Edwin Klebs (1834-1913), Paul Grawitz (1850-1932) y Von Recklinghausen, publicaron un libro conmemorativo (Festschrift Rudolf Virchow zu seinem 71. Geburtstage) (Fig. 5A) ${ }^{17}$. Uno 
de los capítulos fue escrito por Von Recklinghausen bajo el título Die fibröse oder deformirende Ostitis, die Osteomalacie und die osteoplastische Carcinose in ihren gegenseitigen Beziehungen (Osteítis fibrosa o deformante, osteomalacia y calcinosis osteoplástica) (Fig. 5B $)^{17}$. En él, Recklinghausen informó el estudio de autopsia de 16 pacientes cuyos huesos habían sido reemplazados por fibrosis y quistes, a lo que llamó osteítis fibrosa quística (hoy conocida como enfermedad de Von Recklinghausen del hueso; no confundirla con la otra enfermedad de Von Recklinghausen, que es la neurofibromatosis de tipo I). En realidad, Recklinghausen fue muy afortunado en pasar a la historia por esta descripción, porque 50 años después, Fuller Albright (1900-1969), de quien hablaremos en párrafos posteriores, al revisar este manuscrito, llegó a la conclusión de que solo uno de sus 16 pacientes tenía las características clínicas y patológicas típicas del hiperparatiroidismo ${ }^{18,19}$. Albright concluyó que cuatro pacientes habían tenido enfermedad de Paget, tres displasia fibrosa poliostótica y el resto otras afecciones, incluido un carcinoma metastásico en el hueso ${ }^{18,19}$. El único caso que tenía lo que hoy conocemos como osteítis fibrosa fue el de un paciente de 40 años, de nombre $S$. Bleich, que en abril de 1888 se había caído de una escalera sobre su lado izquierdo y fue ingresado al hospital por dolor en la articulación de la cadera ${ }^{19}$. No estaba claro si se trataba de una fractura del cuello del fémur o de una coxitis, y fue tratado con medidas generales y dado de alta. En octubre de ese mismo año, el paciente se resbaló y se fracturó la clavícula, y fue readmitido al hospital, donde estando ingresado se fracturó la diáfisis del fémur derecho, pues se resbaló ¡con el orinal! El dolor continuó y además tenía una deformidad excesiva de varios huesos, y aunque las fracturas parecían sanar, el paciente no podía levantar la pierna. Su mal estado general aumentó y murió el 4 de octubre de 1889. Recklinghausen practicó la autopsia del paciente Bleich e hizo énfasis sobre tres de las principales características patológicas de la enfermedad ósea que tan a menudo acompaña al hiperparatiroidismo: la fibrosis generalizada, los quistes y los tumores pardos (o de células gigantes) (Fig. 5C). Este caso fue diagnosticado como Ostitis de Fibröse Osteosarkome múltiple y fue clasificada como «malacia metaplásica», que se refiere al reemplazo de hueso compacto por osteoide fibroso recién formado. Utilizó la palabra Osteosarkome para referirse a los tumores de color marrón denominados Myeloidtumoren o Riesenzellensarkomen, que posiblemente correspondan a lo que hoy conocemos como «tumor pardo del hiperparatiroidismo». Describió además que el Sr. Bleich tenía «una pequeña glándula rojiza-café en la parte izquierda de la tiroides", lo que parece ser la descripción de una glándula paratiroidea anormal ${ }^{19}$.

Posteriormente se confirmó la relación existente entre trastornos óseos y enfermedad paratiroidea, cuando en 1904 el patólogo alemán Max Askanazy (1865-1940), nacido en Stallupönen, Prusia del Este (hoy Nesterov, Rusia), describió a una mujer de 51 años con cambios óseos de osteítis fibrosa y un tumor paratiroideo, blando, rojo-blanco, de $4.5 \times 2 \times 2 \mathrm{~cm}$, adosado al lóbulo izquierdo de la tiroide ${ }^{20}$. Más tarde, la relación de las glándulas con alteraciones óseas fue corroborada por un patólogo de la Universidad de Viena, Jakob Erdheim (1874-1937), también conocido por la descripción de la enfermedad de Erdheim-Chester y el craneofaringioma (Fig. 6A) ${ }^{21}$. Erdheim demostró, en experimentos con ratas, que la presencia de las glándulas paratiroides era necesaria para el buen crecimiento óseo y dental, e informó que las cuatro glándulas paratiroides estaban crecidas en pacientes con osteomalacia y raquitismo, por lo que dedujo que se trataba de una hiperplasia compensatoria ${ }^{13}$. Dos fueron las observaciones cruciales: 1) la paratiroidectomía producía descalcificación de la dentina de los dientes de las ratas, de modo similar a lo observado en el raquitismo, y 2) todas las paratiroides en el raquitismo espontáneo en las ratas se encontraban crecidas. Erdheim propuso, además, que el crecimiento de las paratiroides que acompaña a los cambios óseos era un fenómeno compensatorio y que la enfermedad ósea debería tratarse con extracto de paratiroides ${ }^{11,13}$. Sin embargo, el patólogo austriaco Friedrich Schlagenhaufer (1866-1930) cuestionó la validez de esta interpretación, pues había observado en dos pacientes con osteomalacia que solo una de las cuatro glándulas estaba crecida, y propuso que los cambios en las glándulas paratiroides eran el evento principal que habían conducido al problema óseo. Por esta razón, le parecía lógico tratar a estos pacientes mediante la extirpación de la glándula enferma ${ }^{11,22}$.

La primera paratiroidectomía en un paciente con enfermedad ósea de Von Recklinghausen fue realizada por Félix Mandl (1892-1957) (Fig. 6B), profesor de cirugía de la Universidad de Viena ${ }^{13,19}$. El paciente Albert Jahne, de 38 años, que era conductor de tranvía, padecía osteítis fibrosa quística grave con fractura femoral y calcio sérico elevado. En 1924 ingresó al Departamento de Cirugía II, del Hospital General de 

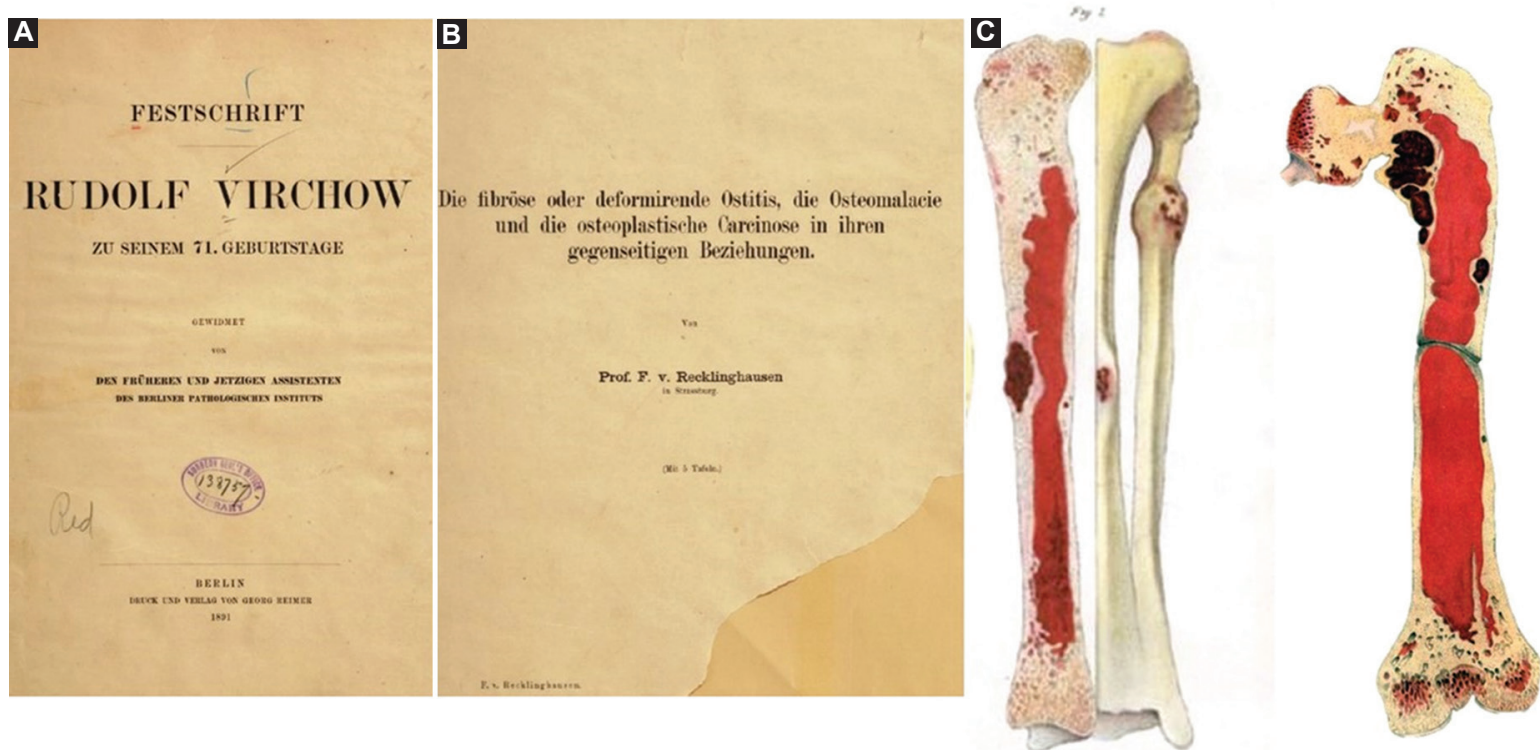

Figura 5. A: publicación del libro conmemorativo Festschrift Rudolf Virchow zu seinem 71. Geburtstage. Fuente: The Virchow "Festschrift". Lancet. 1891;138:1187. B: Die fibröse oder deformirende Ostitis, die Osteomalacie und die osteoplastische Carcinose in ihren gegenseitigen Beziehungen, de Von Recklinghausen, donde describe la osteítis fibrosa quística. C: dibujos del paciente Bleich que muestran las principales características patológicas de la enfermedad ósea que a menudo acompaña al hiperparatiroidismo: fibrosis generalizada, quistes y tumores pardos. Fuente: https://collections.n/m.nih.gov/catalog/n/m:n/muid-62130340R-bk.
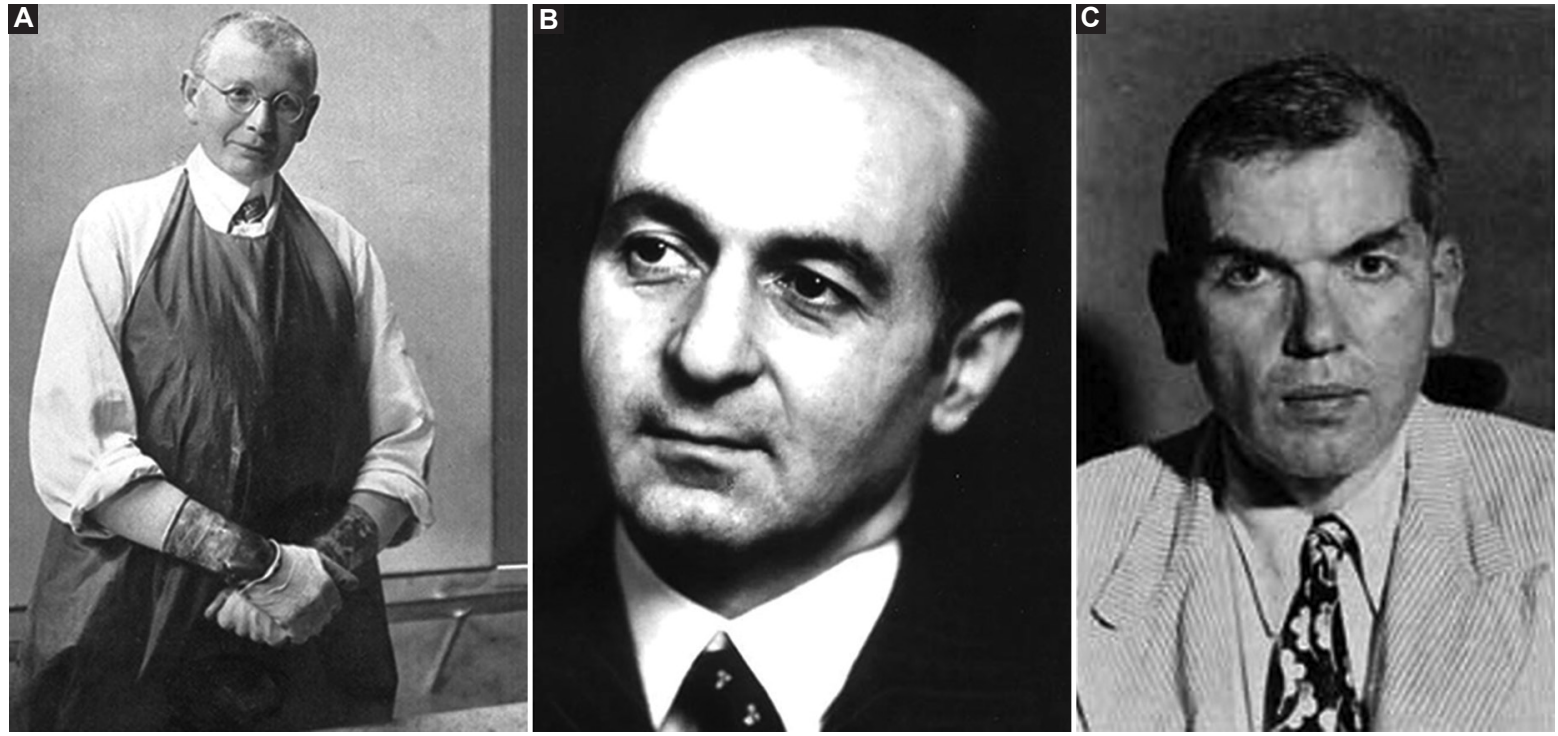

Figura 6. A: Jakob Erdheim (1874-1937). Fuente: https://kehilalinks.jewishgen.org/Drohobycz/families/erdheim.html. B: Félix Mandl (1892-1957). Fuente: https://www.trendsmap.com/twitter/tweet/1235716632376377344. C: Fuller Albright (1900-1969). Fuente: http://collections.countway. harvard.edu/onview/items/show/5897.

Viena, y Mandl pensó que el problema se debía a una deficiencia de paratiroides y fue tratado con tabletas de extracto de paratiroides (tabletas de parathyreodin o extracto de Collip), aceite de hígado de bacalao y baños de lodo, por la posibilidad de que su padecimiento fuera secundario a reumatismo o artritis ${ }^{19}$. En
1925, Albert fue reingresado al hospital y Mandl le trasplantó cuatro glándulas paratiroides procedentes de un hombre que había muerto por accidente, pero no hubo ninguna mejoría, por lo que en desesperación decidió hacerle una paratiroidetcomía, cirugía que nunca había realizado. El 30 de julio de 1925, 
bajo anestesia local, le fue extirpada la glándula paratiroidea inferior izquierda, y se describió que era amarilla-café, de $25 \times 15 \times 12 \mathrm{~mm}$ (las dimensiones normales son $5 \times 3 \times 1 \mathrm{~mm}$ ) y que estaba adherida al nervio recurrente. Las otras tres glándulas paratiroides eran normales. El paciente mejoró en forma espectacular, los huesos ya no le dolían y las concentraciones de calcio sérico mejoraron. Mandl había demostrado por primera vez que la hipótesis de Schlagenhaufer era correcta, es decir, que la enfermedad ósea era secundaria a una lesión de la glándula paratiroidea. «Fue un experimento con resultados afortunados", indicó Mandl, y pasó a la historia como el primer cirujano en haber resecado un tumor paratiroideo, y Albert como el primer paciente que había sido curado de un tumor paratiroideo ${ }^{23}$.

La glándula extirpada fue estudiada por Erdheim, quien describió que estaba compuesta por:

«... hebras alveolares o plexiformes y cohesivas que estaban separadas entre sí por hebras estrechas de tejido conectivo pobre en células. Las células epiteliales que componen los alvéolos yacían una al lado de la otra como un mosaico, separadas por líneas finas y rojas, y tenían un protoplasma que a veces era transparente y con un núcleo aparentemente libre, a veces parecía estar lleno de partículas finas y gránulos eosinofílicos. El núcleo era generalmente redondo, vesicular, brillante y de tamaño variable. Un núcleo puede ser cinco veces más grande que otro, pero rara vez se encuentran aquellos de tamaño excesivo. Donde el epitelio estaba directamente sobre el estroma, a veces mostraba una disposición en empalizada... No se puede encontrar tejido paratiroideo normal en el corte, en ningún lugar... Se puede suponer un tumor maligno debido al tamaño inconstante del núcleo celular y las figuras mitóticas y el polimorfismo de las células...»

Años más tarde, Mandl consideró que este tumor era un «adenoma» ${ }^{3}$.

El paciente Albert pudo caminar con la ayuda de un bastón, y el dolor pélvico y de extremidades desapareció. Sin embrago, 6 años después tuvo una recurrencia y en septiembre de 1932 presentó un cálculo renal, nuevamente hipercalcemia, descenso de la fosfatasa en sangre, los huesos se descalcificaron y quedó una vez más incapacitado. Mandl volvió a operar a Albert el 18 de octubre de 1933, pero no encontró ningún tumor en toda la región del cuello y en el mediastino, y extrajo dos glándulas paratiroides normales. El paciente falleció en febrero de 1936 y la autopsia no reveló tumor paratiroideo alguno. Varios huesos presentaban cambios secundarios al hiperparatiroidismo grave y ambos riñones tenía nefrocalcinosis $^{3}$. Se supone que Albert murió de un carcinoma paratiroideo metastásico, pero es algo que nunca podrá ser resuelto ${ }^{24}$. Dos años después de la cirugía, Mandl recibió el Venia Legendi, que es la más alta calificación académica para ser profesor de cirugía en la Universidad de Viena, misma que le fue retirada por el régimen nazi por el hecho de ser judío. Tuvo que salir de Viena y continúo su práctica quirúrgica en el Hospital Haddassah, en Jerusalén, y regresó a Viena ya acabada la Segunda Guerra Mundial, en 1947, cuando fue nombrado jefe de cirugía del Kaiser Franz Josef Spital. Murió a los 65 años de edad por complicación de una influenza ${ }^{13,24}$.

Como vimos, el paciente Albert recibió el extracto paratiroideo que Collip y Hanson habían aislado años antes. El primer informe del aislamiento de un extracto paratiroideo efectivo es el de Adolph Melanchthon Hanson (1880-1959), en 1923, cuando aún era estudiante de medicina en la Universidad de Minnesota. Hanson, quien había sido alumno de Harvey W. Cushing (1869-1939), desarrolló un método para extraer hormona paratiroidea de las glándulas paratiroides bovinas para el tratamiento de la tetania, en un laboratorio acondicionado en su casa ${ }^{25}$. Por el mismo periodo de tiempo, el bioquímico canadiense James B. Collip (1892-1965) aisló de las glándulas paratiroides una sustancia que llamo parathyrin, y estaba convencido de que las glándulas paratiroides contenían una hormona reguladora del calcio ${ }^{26}$. El método utilizado por ambos para extraer la hormona fue similar. Trituraron las paratiroides de un buey y las trataron con ácido clorhídrico diluido, que luego se concentró por vaporación y tituló a pH neutro, con lo que aislaron la paratohormona ${ }^{25,26}$. Collip es más conocido por haber participado en el aislamiento de la insulina (aunque el comité del Premio Nobel no lo consideró merecedor de este) que por su papel en el descubrimiento de la hormona paratiroidea, una proeza de igual importancia. La hormona paratiroidea fue purificada en 1959 por Howard Rasmussen (19251999) y Lyman C. Craig (1906-1974) del Instituto Rockefeller, en Nueva York, y su estructura peptídica también fue definida por ellos en $1962^{27}$. Craig recibió el premio Albert Lasker en 1963 y fue nominado en 29 ocasiones para el Premio Nobel en Química.

Finalmente, un inmunoensayo para la medición de diversas hormonas, entre ellas la hormona paratiroidea, fue desarrollado en colaboración por Solomon A. Berson (1919-1972) y Rosalyn S. Yalow (1921-2011) 
en 1963, lo que valió a Yalow el Premio Nobel en Medicina o Fisiología en 1977 (Berson no recibió el premio debido a que había muerto de un infarto al corazón a la prematura edad de 53 años en 1972, y no se otorgan premios Nobel póstumos) ${ }^{28,29}$.

En el avance de los conceptos actuales en fisiología de las glándulas paratiroides no puede ser omitido Fuller Albright, quien dedicó casi toda su vida académica al estudio de las paratiroides (Fig. 6C) ${ }^{30}$. Albright nació en Búfalo, Nueva York, el 12 de enero de 1900, y estudió medicina en la Universidad de Harvard. Al terminar el internado, realizó primero un año de investigación sobre el metabolismo de calcio con el profesor Joseph C. Aub (1890-1973), luego una rotación en la Universidad Johns Hopkins bajo la tutela del profesor Warfield T. Langcope (1877-1953), y después pasó 1 año al lado de Erdheim en Viena; estas tres personalidades fueron una gran inspiración para su posterior interés en el estudio de las paratiroides ${ }^{30,31}$.

A su regreso de Viena, Albright se instaló en el Hospital General de Massachusetts y organizó el área de endocrinología. Su trabajo fue muy productivo y describió numerosas enfermedades, como la displasia fibrosa poliostótica asociada a manchas cutáneas de color café con leche y pubertad precoz (síndrome de McCune-Albright), la nefrocalcinosis con acidosis hiperclorémica (síndrome de Lightwood-Albright), el síndrome amenorrea-galactorrea por prolactinoma hipofisario (síndrome de Forbes-Albright), la importancia de la menopausia en la osteoporosis, las diversas formas de hiperplasia suprarrenal congénita y los diferentes tipos de hiperparatiroidismo, entre muchas otras contribuciones originale ${ }^{30}$. Su predicción, hacia 1945, del eventual control de la natalidad secundario a la terapia hormonal, se conoce como «la profecía de Albright»30,31.

Mientras Albright era aún estudiante de medicina inició su afición por el estudio de las glándulas paratiroides. Desde el año en que presentó el trabajo titulado The Physiology and Physiological Pathology of Calcium y hasta su muerte en 1969 a causa de una complicación de palidotomía por la enfermedad de Parkinson, Albright dedicó casi toda su carrera al estudio de estas glándulas. A la edad de 36 años fue diagnosticado con enfermedad de Parkinson, y en 1956, en contra de la opinión de muchos médicos, decidió someterse a una palidotomía. Desafortunadamente, el procedimiento quirúrgico se complicó con una hemorragia, que lo dejó afásico y comatoso durante los 13 años restantes de su vida, durante los cuales fue atendido en el Hospital General de Massachusetts ${ }^{31}$.

\section{Conclusiones}

Poco más de 150 años han pasado desde que las descripciones de Owen, Sandström y Gley dieran pie a un sinfín de estudios sobre la ultraestructura, la bioquímica, la función y la patología de las glándulas paratiroides. Los nombres de sus descubridores han sido poco a poco olvidados, pero sin duda merecen ser por siempre recordados. El término «paratiroides» es posiblemente poco preciso, pues no siempre son para-, sino que indistintamente pueden ser intra-, epi- o extra- en relación con la tiroides. Además, únicamente guardan con esta una relación anatómica, pero no tienen ninguna conexión fisiológica. Estas glándulas históricamente han recibido nombres como glandules thyréoïdennes, glandules thymiques, glandules branchiales o parathymus III, IV. Por desgracia, muchos epónimos han desaparecido, ya que estos no aportan nada al significado y la función del órgano en cuestión, pero para aquellos que todavía usan los epónimos, las paratiroides cuentan con el mejor nombre: «glándulas de Owen-Sandström-Gley».

\section{Financiamiento}

Este trabajo no recibió financiamiento.

\section{Conflicto de intereses}

El autor declara no tener conflicto de intereses.

\section{Responsabilidades éticas}

Protección de personas y animales. Los autores declaran que para esta investigación no se han realizado experimentos en seres humanos ni en animales.

Confidencialidad de los datos. Los autores declaran que han seguido los protocolos de su centro de trabajo sobre la publicación de datos de pacientes.

Derecho a la privacidad y consentimiento informado. Los autores declaran que en este artículo no aparecen datos de pacientes.

\section{Bibliografía}

1. Erraez-Jaramillo PJ, Ortiz-Hidalgo C. Anatomía microscópica de las glándulas paratiroides normal. Principios generales para residentes de endocrinología y patología, con una breve nota histórica. Rev Mex Endocrinol Metab Nutr. 2020;7:43-53.

2. van der Walt J. Pathology of the parathyroid glands. Diag Histopathol. 2012;18:221-33.

3. Carney JA. The glandulae parathyroideae of Ivar Sandström. Contributions from two continents. Am J Surg Pathol. 1996;20:1123-44. 
4. Eknoyan G. A history of the parathyroid glands. Am J Kidney Dis. 1995;26:801-7.

5. McAneny D, Beazley RM. The glands of Owen. Who was Owen? Surgery. 2016;159:7-10.

6. Modarai B, Sawyer A, Ellis H. The glands of Owen. J R Soc Med. 2004;97:494-5.

7. Torrens $H$. When did the dinosaur get its name? New Sci. 1992:134:40-4.

8. Wessels Q, Taylor AM. Anecdotes to the life and times of Sir Richard Owen (1804-1892) in Lancaster. J Med Biogr. 2017;25:226-33.

9. García Bartual M. ¿Fue Richard Owen un villano de la ciencia? Disponible en: https://www.lavanguardia.com/historiayvida/historia-contemporanea/20170620/47313745809/fue-richard-owen-un-villano-de-la-ciencia.html

10. Owen R. On the Anatomy of the Indian Rhinoceros (Rh. unicornis, L.). Zool Soc. 1852;IV:31-58.

11. Johansson H. The Uppsala anatomist Ivar Sandström and the parathyroid gland. Upsala J Med Sci. 2015;120:72-7.

12. Sandström IV. On a new gland in man and several mammals - glandulae parathyroideae. Upsala Läk Förenings Förh. 1879-80;15:441-71.

13. Vermeulen AHM. The birth of endocrine pathology. How Erdheim misunderstood parathyroids. Virchows Arch. 2010;457:283-90.

14. Breimer L, Sourander P. The discovery of the parathyroid glands in 1877 triumph and tragedy of Ivar Sandström. Bull Hist Med. 1981;55:558-63.

15. Thomson RL. A study of parathyroid glands in paralysis agitans. J Med Res. 1906;15:399-424-5.

16. Mac Callum $\mathrm{G}$, Vogetlin $\mathrm{C}$. On the relation of tetany to the parathyroid glands and to calcium metabolism. J Exp Med. 1909;11:118-51.

17. Editorial. The Virchow "Festschrift". Lancet. 1891;138:1187.

18. Cherian ET, Guttenberg KB. Osteitis fibrosa cystica. QJM. 2018;111:487.
19. Albright F. A page out of the history of hyperparathyroidism. J Clin Endocrinol. 1948;8:637-57.

20. Askanasy M. Uber Ostitis deformans ohne ostoides gewebe. Arb Geb Path Anat Inst Tubingen. 1903;4:398-422.

21. Pascual JM, Rosdolsky M, Prieto R, Strau $\beta$ S, Winter E, Ulrich W. Jakob Erdheim (1874-1937): father of hypophyseal-duct tumors (craniopharyngiomas). Virchows Arch. 2015;467:459-69.

22. Schlagenhaufer F. Über diffuse ossifizierende Periostitis. Ztsch f Heilk. Abt pathol Anat. 1904;25:364-80.

23. Nordenström J, Marcus R. History of the parathyroid gland and their secretory product. Parathyroid hormone. En: Biliezikian J, editor. The parathyroid gland. Basic and clinical concepts. $3^{\text {rd }}$ ed. Amsterdam: Elsevier; 2015. p. 1-17.

24. Niederle BE, Schmidt G, Organ CH, Niederle B. Albert $\mathrm{J}$ and his surgeon: a historical reevaluation of the first parathyroidectomy. J Am Coll Surg. 2006;202:181-90.

25. Hanson AM. An elementary chemical study of the parathyroid glands of cattle. Mil Surg. 1923;53:280-4.

26. Collip JB. The extraction of parathyroid hormone which will prevent or control parathyroid tetany and which regulates the level of blood calcium. J Biol Chem. 1925;63:395-438.

27. Rasmussen H, Craig LC. Purification of parathormone by use of countercurrent distribution. J Am Chem Soc. 1959;81:5003.

28. Friedman A. Remembrance: the Berson and Yalow saga. J Clin Endocrinol Metab. 2002;87:1925-8.

29. The Endocrine Society Staff. In Memoriam: Dr. Rosalyn Yalow, PhD., 1921-2011. Mol Endocrinol. 2012;26:713-4.

30. Kleeman CR, Levine BS, Felsenfeld AF. Fuller Albright: the consummate clinical investigator. Clin J Am Soc Nephrol. 2009;4:1541-1546.

31. River V. Dr. Fuller Albright. Biografías Médicas Ilustradas. Disponible en: https://biografiasmedicasilustradas.blogspot.com/2015/06/dr-fuller-albright.html 